\title{
Anesthesia for Guillain-Barré Syndrome Do we need neuromuscular blocking agents? \\ A case report
}

Marques F.V ${ }^{1}$; Norte G. ${ }^{1}$, Madeira F. ${ }^{1}$, Lapa T.A. ${ }^{1,2}$, Viana J.S. ${ }^{1,2}$

${ }^{1}$ Coimbra Hospital and University Centre, Anesthesiology Service, Coimbra, Portugal;

${ }^{2}$ University of Beira Interior, Portugal, Faculty of Health Sciences, Covilhã, Portugal

Background: Guillain-Barré (GB) syndrome is a challenge for the anesthesiologist. Our purpose is to report and discuss the anesthesia of a GB patient for major orthopedic surgery, managed without neuromuscular blocking agents.

\section{Case report:}

Male, 43 years-old, ASA II, proposed for a total hip replacement.

D History of paralysis caused by GB 4 years ago with partial recovery after therapy.

$>$ On admission, moderate muscular weakness without apparent respiratory compromise.

$>$ After anesthesia induction with fentanyl $100 \mu \mathrm{g}$ and propofol 200mg we inserted an orotracheal tube without difficulties. During surgery we administered desflurane 4.1 to $6.0 \%$ endtidal, fentanyl $200 \mu \mathrm{g}$, paracetamol $1 \mathrm{~g}$ and parecoxib $40 \mathrm{mg}$.

> Surgery lasted for 2 hours and was accomplished uneventfully.

> Patient was hemodynamically stable (Figure 1) and the tracheal tube was removed at the end of the surgery without complications.

After 2 hours the patient was recovered without incidents.

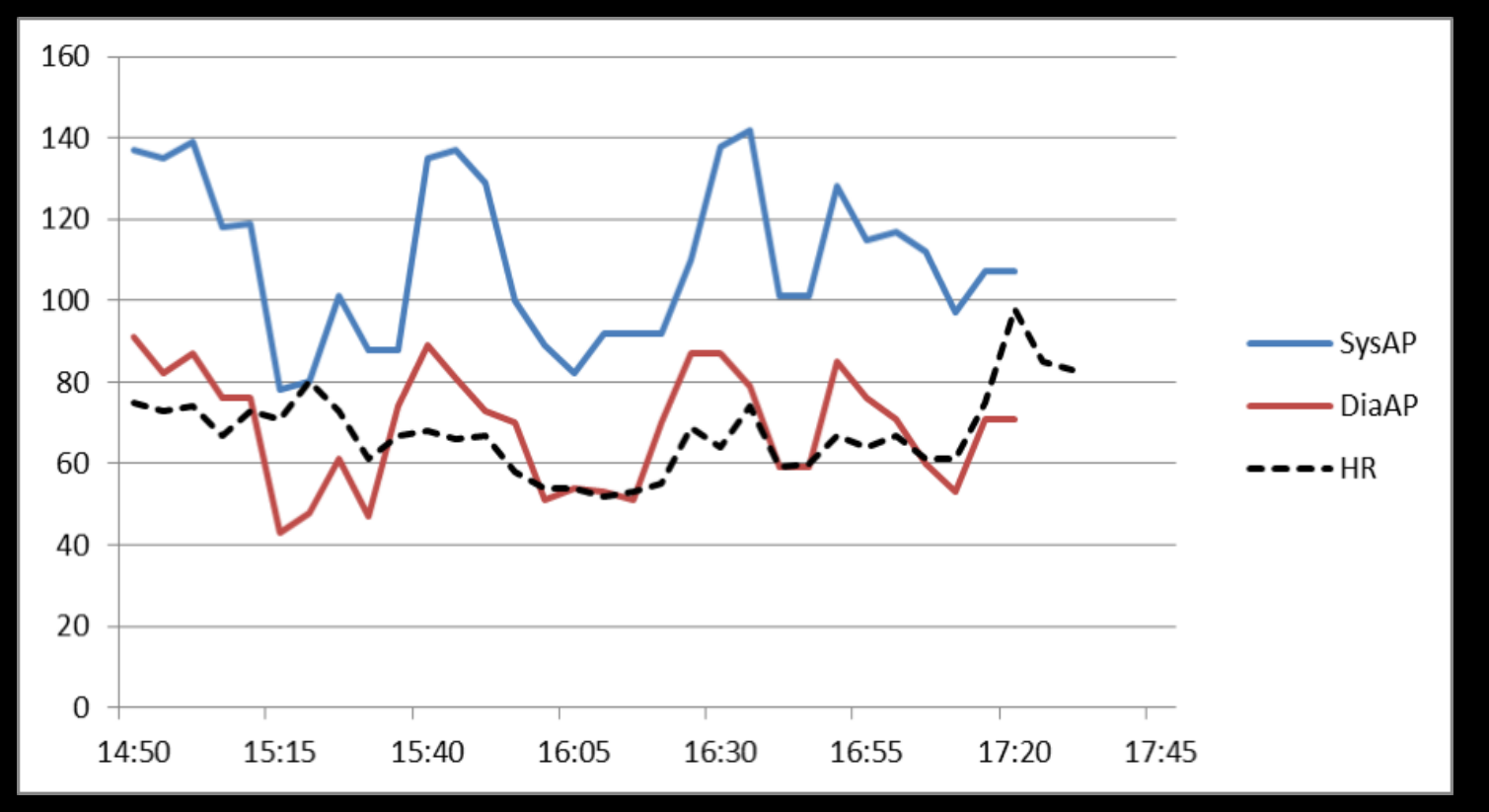

Figure 1

\section{Discussion:}

Our case shows that a GB patient can be successfully submitted to major orthopedic surgery under general anesthesia without any neuromuscular blockers.

Previous reports of anesthesia in GB stated three points:

$\checkmark$ First, the potential of neuroaxial anesthesia to worsen the disease,

$\checkmark$ Second, the risks of hyperkalemia and cardiac arrest with succinylcholine,

$\checkmark$ Third, the increased sensivity of these patients to non-depolarizing drugs [1-2].

As neuromuscular blockers are not absolutely precluded, a point that is usually missed is why patients with muscular weakness need relaxants.

$>$ As far as we know, in evidence-based anesthesia, we do not find any support to use relaxants in GB.

$>$ Also, our case also suggests its possible futility in this subpopulation of patients.

$>$ In consequence we would recommend its use only as rescue therapy and only in cases where muscular function would be enough to produce difficult surgical conditions.

Learning Points: In GB patients, depolarizing drugs have the risk of hyperkalemia, and non-depolarizing drugs are associated with prolonged blockage and post-operative ventilatory support. What evidence supports the need of neuromuscular blockers during general anesthesia for GB? Our case suggests that we may not need neuromuscular blockers to anesthetize GB patients with muscular weakness.

1. Jacob AK, Kopp SL. Regional anesthesia in the patient with preexisting neurologic disorders. Adv Anesth. 2011;29:1-18.

2. Succinylcholine-induced Hyperkalemia in Acquired Pathologic states: Etiologic Factors and Molecular Mechanisms. The Journal of the American Society of Anesthesiologists.
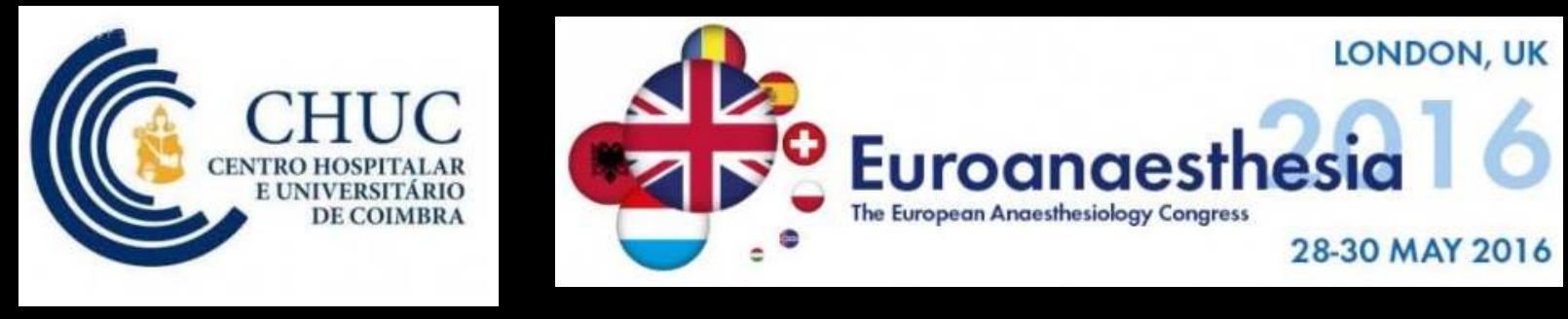